\title{
A Class of Nonlocal Coupled Semilinear Parabolic System with Nonlocal Boundaries
}

\author{
Hong Liu' ${ }^{1}$ and Haihua $\mathrm{Lu}^{2}$ \\ ${ }^{1}$ Teaching Group of Mathematics, Zhenjiang Watercraft College of PLA, Zhenjiang 212003, China \\ ${ }^{2}$ School of Science, Nantong University, Nantong 226007, China
}

Correspondence should be addressed to Haihua Lu; haihualu@ntu.edu.cn

Received 30 August 2013; Accepted 1 December 2013; Published 16 February 2014

Academic Editors: Q. Liu, M. Wang, and P. Wang

Copyright (C) $2014 \mathrm{H}$. Liu and H. Lu. This is an open access article distributed under the Creative Commons Attribution License, which permits unrestricted use, distribution, and reproduction in any medium, provided the original work is properly cited.

We investigate the positive solutions of the semilinear parabolic system with coupled nonlinear nonlocal sources subject to weighted nonlocal Dirichlet boundary conditions. The blow-up and global existence criteria are obtained.

\section{Introduction}

In this paper, we consider the positive solutions of the semilinear parabolic system with coupled nonlinear nonlocal sources subject to weighted nonlocal Dirichlet boundary conditions:

$$
\begin{gathered}
u_{i t}=\Delta u_{i}+\int_{\Omega} u_{i}^{q_{i}} u_{i+1}^{p_{i}}(x, t) \mathrm{d} x, \\
i=1,2, \ldots, k, \quad u_{k+1}=u_{1}, \quad x \in \Omega, \quad t>0, \\
u_{i}(x, t)=\int_{\Omega} \varphi_{i}(x, y) u_{i}(y, t) \mathrm{d} y, \\
i=1,2, \ldots, k, \quad x \in \partial \Omega, \quad t>0, \\
u_{i}(x, 0)=u_{i, 0}(x), \quad i=1,2, \ldots, k, \quad x \in \Omega,
\end{gathered}
$$

where $\Omega$ is a bounded domain in $\mathbb{R}^{N}, N \geq 1$, with smooth boundary $\partial \Omega$. The exponents $p_{i}>0, q_{i} \geq 0$. The weighted functions $\varphi_{i}$ in the boundary conditions are continuous, nonnegative on $\partial \Omega \times \bar{\Omega}$ and $\int_{\Omega} \varphi_{i}(x, y) \mathrm{d} y>0$ on $\partial \Omega$. The

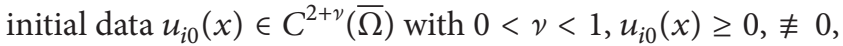
and satisfy the compatibility conditions.

Many physical phenomena were formulated into nonlocal mathematical models and studied by many authors [1-13]. For example, in [1], Bebernes and Bressan studied an ignition model for a compressible reactive gas which is a nonlocal reaction-diffusion equation. Furthermore, Bebernes et al. [14] considered a more general model:

$$
\begin{gathered}
u_{t}-\Delta u=f(u)+g(t), \quad x \in \Omega, t>0, \\
u(x, 0)=u_{0}(x), \quad x \in \Omega, \\
u(x, t)=0, \quad x \in \partial \Omega, t>0,
\end{gathered}
$$

where $u_{0}(x) \geq 0, g(t)>0$ or $g(t)=(k /|\Omega|) \int_{\Omega} u_{t}(x, t) d x$ with $k>0$. Chadam et al. [15] studied another form of (2) with $f(u)=0$ and $g(t)=\int_{\Omega} \psi(u(x, t)) \mathrm{d} x$ and proved that the blow-up set is the whole region (including the homogeneous Neumann boundary conditions). Souplet $[16,17]$ considered (2) with the general function $g(t)$. Pao [18] discussed a nonlocal reaction-diffusion equation arising from the combustion theory.

The problems with both nonlocal sources and nonlocal boundary conditions have been studied as well. To motivate our study, we give a short review of examples of such parabolic equations or systems studied in the literature. For example, Lin and Liu [19] studied the following problem:

$$
\begin{gathered}
u_{t}-\Delta u=\int_{\Omega} f(u(y, t)) \mathrm{d} y, \quad x \in \Omega, t>0, \\
u(x, t)=\int_{\Omega} \varphi(x, y) u(y, t) \mathrm{d} y, \quad x \in \partial \Omega, t>0, \\
u(x, 0)=u_{0}(x), \quad x \in \Omega ;
\end{gathered}
$$


they established local existence, global existence, and nonexistence of solutions and discussed the blow-up properties of solutions. form

Gladkov and Kim [20] considered the problem of the

$$
\begin{gathered}
u_{t}=\Delta u+c(x, t) u^{p}, \quad x \in \Omega, t>0, \\
u(x, t)=\int_{\Omega} \varphi(x, y) u^{l}(y, t) \mathrm{d} y, \quad x \in \partial \Omega, t>0, \\
u(x, 0)=u_{0}(x), \quad x \in \Omega,
\end{gathered}
$$

with $p, l>0$. And some criteria for the existence of global solution as well as for the solution to blow up in finite time were obtained.

In [21], Kong and Wang studied system (1) when $k=2$ :

$$
\begin{gathered}
u_{t}=\Delta u+\int_{\Omega} u^{m}(x, t) v^{n}(x, t) \mathrm{d} x, \quad x \in \Omega, t>0, \\
v_{t}=\Delta v+\int_{\Omega} u^{p}(x, t) v^{q}(x, t) \mathrm{d} x, \quad x \in \Omega, t>0, \\
u(x, t)=\int_{\Omega} \varphi(x, y) u(y, t) \mathrm{d} y, \\
v(x, t)=\int_{\Omega} \psi(x, y) v(y, t) \mathrm{d} y, \\
x(x \in \partial \Omega, t>0, \\
u(x, 0)=u_{0}(x), \quad v(x, 0)=v_{0}(x), \quad x \in \Omega
\end{gathered}
$$

they obtained the following results, and we extend them as follows.

(i) Assume that $m, q<1$ and $n p \leq(1-m)(1-q)$ hold; then the solution of (5) exists globally.

(ii) If one of the following conditions holds:

$$
\begin{aligned}
& \text { (a) } m>1 \\
& \text { (b) } q>1 \\
& \text { (c) } n p>(1-m)(1-q)
\end{aligned}
$$

then the solution of (5) blows up in a finite time for the sufficiently large initial data.

(iii) Assume that $\int_{\Omega} \varphi(x, y) \mathrm{d} y \geq 1$ and $\int_{\Omega} \psi(x, y) \mathrm{d} y \geq$ 1 for all $x \in \partial \Omega$ and one of (6) holds; then the solution of problem (5) blows up in a finite time for any positive initial data.

Recently, Zheng and Kong [22] also studied the following problem:

$$
\begin{aligned}
& u_{t}-\Delta u=u^{m}(x, t) \int_{\Omega} v^{n}(x, t) \mathrm{d} x, \quad x \in \Omega, t>0, \\
& v_{t}-\Delta v=v^{q}(x, t) \int_{\Omega} u^{p}(x, t) \mathrm{d} x, \quad x \in \Omega, t>0,
\end{aligned}
$$

with the same initial and boundary conditions as (5), and they established similar conditions for global and nonglobal solutions and also blow-up solutions.

The main purpose of this paper is to get the blow-up criterion of problem (1) for any positive integer $k$.

In the following, we set $Q_{T}=\Omega \times(0, T)$, and $S_{T}=\partial \Omega \times$ $(0, T)$ with $0<T<\infty$ for convenience.

It is known by the standard theory $[16,23]$ that there exists a local positive solution to (1). Moreover, by the comparison principle (see Lemma 10 in the next section), the uniqueness of solutions holds if $p_{i}, q_{i} \geq 1, i=1,2, \ldots, k$.

Theorem 1. Problem (1) has a positive classical solution $\left(u_{1}, u_{2}, \ldots, u_{k}\right) \in\left[C^{2+\widehat{\alpha}, 1+\widehat{\alpha} / 2}\left(Q_{T}\right) \cap C\left(\bar{Q}_{T}\right)\right]^{k}$ for some $\widehat{\alpha}: 0<$ $\widehat{\alpha}<1$. Moreover, if $T<\infty$, then

$$
\lim _{t \rightarrow T}\left(\left\|u_{1}(\cdot, t)\right\|_{\infty}+\cdots+\left\|u_{k}(\cdot, t)\right\|_{\infty}\right)=\infty
$$

Theorem 2. If exponents $p_{i}, q_{i}, i=1,2, \ldots, k$ satisfy

$$
\begin{gathered}
q_{i}<1, \quad i=1,2, \ldots, k, \\
p_{1} p_{2} \cdots p_{k} \leq\left(1-q_{1}\right)\left(1-q_{2}\right) \cdots\left(1-q_{k}\right),
\end{gathered}
$$

the solution $\left(u_{1}, u_{2}, \ldots, u_{k}\right)$ of (1) exists globally for any nontrivial nonnegative initial data.

Theorem 3. If exponents $p_{i}, q_{i}, i=1,2, \ldots, k$ satisfy one of the following:

$$
\begin{aligned}
& \text { (a) } q_{r}>1, \quad r \in\{1,2, \ldots, k\}, \\
& \text { (b) } p_{1} p_{2} \cdots p_{k}>\left(1-q_{1}\right)\left(1-q_{2}\right) \cdots\left(1-q_{k}\right)
\end{aligned}
$$

and if $\int_{\Omega} \varphi_{i}(x, y) \mathrm{d} y<1, i=1,2, \ldots, k$, for all $x \in \partial \Omega$, then the solution of (1) exists globally for small nonnegative initial data.

Theorem 4. If exponents $p_{i}, q_{i}, i=1,2, \ldots, k$ satisfy one of the following:

$$
\begin{aligned}
& \text { (a) } q_{r}>1, \quad r \in\{1,2, \ldots, k\}, \\
& \text { (b) } p_{1} p_{2} \cdots p_{k}>\left(1-q_{1}\right)\left(1-q_{2}\right) \cdots\left(1-q_{k}\right),
\end{aligned}
$$

then the solution of (1) blows up in finite time for large initial data.

If the initial data $u_{i, 0}(x)$ satisfies

$$
\text { (H) } \Delta u_{i, 0}+\int_{\Omega} u_{i, 0}^{q_{i}} u_{i, 0+1}^{q_{i}} \geq 0, \quad i=1,2, \ldots, k,
$$

we have another blow-up result.

Theorem 5. Assume that

$$
q_{r}>1, \quad \int_{\Omega} \varphi_{r}(x, y) \mathrm{d} y \geq 1, \quad r \in\{1,2, \ldots, k\}
$$

and the condition $(H)$ holds. Then the solution of (1) blows up in finite time for any positive initial data. 
This paper is organized as follows. Section 2 is devoted to some comparison principles. In Section 3, we prove two global existence results. The blow-up results are proved in the final section.

\section{Comparison Principle}

Before proving the main results, we give the maximum and comparison principles related to the problem. First, we give the following definition of the upper and lower solutions.

Definition 6. A pair of functions $\left(\bar{u}_{1}(x, t), \ldots, \bar{u}_{k}(x, t)\right)$ is called an upper solution of (1), if, for every $i=$ $1,2, \ldots, k, \bar{u}_{i}(x, t) \in C^{2,1}\left(Q_{T}\right) \cap C\left(\bar{Q}_{T}\right)$ and satisfies

$$
\begin{gathered}
\bar{u}_{i t} \geq \Delta \bar{u}_{i}+\int_{\Omega} \bar{u}_{i}^{q_{i}} \bar{u}_{i+1}^{p_{i}}(x, t) \mathrm{d} x, \quad \bar{u}_{k+1}=\bar{u}_{1}, \\
x \in \Omega, t>0, \\
\bar{u}_{i}(x, t) \geq \int_{\Omega} \varphi_{i}(x, y) \bar{u}_{i}(y, t) \mathrm{d} y, \quad x \in \partial \Omega, t>0, \\
\bar{u}_{i}(x, 0) \geq u_{i, 0}(x), \quad x \in \Omega .
\end{gathered}
$$

Similarly, a lower solution of (1) is defined by the opposite inequalities.

Lemma 7. Suppose that $a_{i j}, b_{i}, f_{i} \in C\left(\bar{Q}_{T}\right)$ and $f_{i} \geq 0, c_{i}$, $d_{i} \geq 0$ in $Q_{T}, g_{i}(x, y) \geq 0$ on $\partial \Omega \times \bar{\Omega}, \int_{\Omega} g_{i}(x, y) \mathrm{d} y>0$ on $\partial \Omega, i=1,2, \ldots, k, j=1,2, \ldots, N$. If, for every $i=1,2, \ldots, k$, $w_{i} \in C^{2,1}\left(Q_{T}\right) \cap C\left(\bar{Q}_{T}\right)$ and satisfies

$$
\begin{aligned}
w_{i t}-\Delta w_{i} \geq \sum_{j=1}^{N} a_{i j} \frac{\partial w_{i}}{\partial x_{j}}+b_{i} w_{i} & \\
+ & f_{i}(x, t) \int_{\Omega}\left(c_{i} w_{i}+d_{i} w_{i+1}\right) \mathrm{d} x, \quad(x, t) \in Q_{T}, \\
w_{i}(x, t) \geq \int_{\Omega} g_{i}(x, y) w_{i}(y, t) \mathrm{d} y, \quad(x, t) \in S_{T}, & \\
& w_{i}(x, 0)>0, \quad x \in \Omega,
\end{aligned}
$$

where $w_{k+1}=w_{1}$, then $w_{i}(x, t)>0, i=1,2$, on $\bar{Q}_{T}$.

Proof. Set $\bar{b}_{i}=\sup _{\overline{\mathrm{Q}}_{T}}\left|b_{i}\right|, z_{i}=e^{-K t} w_{i}$ with $K>\max \left\{\bar{b}_{i}, i=\right.$ $1,2, \ldots, k\}$. Then

$$
\begin{gathered}
z_{i t}-\Delta z_{i}+\left(K-b_{i}\right) z_{i} \\
\geq \sum_{j=1}^{N} a_{i j} \frac{\partial z_{i}}{\partial x_{j}}+f_{i}(x, t) \int_{\Omega}\left(c_{i} z_{i}+d_{i} z_{i+1}\right) \mathrm{d} x, \quad(x, t) \in Q_{T}, \\
z_{i}(x, t) \geq \int_{\Omega} g_{i}(x, y) z_{i}(y, t) \mathrm{d} y, \quad(x, t) \in S_{T}, \\
z_{i}(x, 0)>0, \quad x \in \Omega .
\end{gathered}
$$

Since $z_{i}(x, 0)>0, i=1,2, \ldots$, there exists $\delta>0$ such that $z_{i}>$ 0 for $(x, t) \in \bar{\Omega} \times(0, \delta)$. Suppose for a contradiction that $\bar{t}=$ $\sup \left\{t \in(0, T): z_{i}>0\right.$ on $\left.\bar{\Omega} \times[0, t), i=1,2, \ldots, k\right\}<T$. Then $z_{i} \geq 0$ on $\bar{Q}_{\bar{t}}$, and at least one of $z_{i}$ vanishes at $(\bar{x}, \bar{t})$ for some $\bar{x} \in \bar{\Omega}$. Without loss of generality, suppose that $z_{1}(\bar{x}, \bar{t})=0=$ $\inf _{\overline{Q_{\bar{t}}}} z_{1}$. If $(\bar{x}, \bar{t}) \in Q_{\bar{t}}$; by virtue of the first inequality of (16), we find that

$$
z_{1 t}-\Delta z_{1}+\left(K-b_{1}\right) z_{1}-\sum_{j=1}^{N} a_{i j} \frac{\partial z_{i}}{\partial x_{j}} \geq 0, \quad(x, t) \in \bar{Q}_{\bar{t}} .
$$

This leads to the conclusion that $z_{1} \equiv 0$ in $\bar{Q}_{\bar{t}}$ by the strong maximum principle, a contradiction. If $(\bar{x}, \bar{t}) \in S_{\bar{t}}$, this results in a contradiction too, that

$$
0=z_{1}(\bar{x}, \bar{t})=\int_{\Omega} g_{1}(x, y) z_{1}(y, t) \mathrm{d} y>0
$$

due to $\int_{\Omega} g_{1}(x, y) \mathrm{d} y>0$ on $\partial \Omega$. This proves that $z_{1}>0$ and consequently $w_{1}>0$. We complete the proof.

Lemma 8. Suppose that, for every $i=1,2, \ldots, k, w_{i} \in$ $C^{2,1}\left(Q_{T}\right) \cap C\left(\bar{Q}_{T}\right)$ and satisfies

$$
\begin{gathered}
w_{i t}-\Delta w_{i} \geq \int_{\Omega}\left(a_{i}(x, t) w_{i}+b_{i}(x, t) w_{i+1}\right) \mathrm{d} x, \quad(x, t) \in Q_{T}, \\
w_{i}(x, t) \geq \int_{\Omega} g_{i}(x, y) w_{i}(y, t) \mathrm{d} y, \quad(x, t) \in S_{T}, \\
w_{i}(x, 0) \geq 0, \quad x \in \Omega,
\end{gathered}
$$

where $w_{k+1}=w_{1}$ and $a_{i}(x, t), b_{i}(x, t)$ are continuous, nonnegative functions in $\bar{Q}_{T}, g_{i}(x, y) \geq 0$ on $\partial \Omega \times \bar{\Omega}$ such that $\int_{\Omega} g_{i}(x, y) \mathrm{d} y<1$ on $\partial \Omega$, and there exist positive constants $C_{i}$ such that $\int_{\Omega}\left(a_{i}(x, t)+b_{i}(x, t)\right) \mathrm{d} x \leq C_{i}$. Then $w_{i}(x, t) \geq 0, i=$ 1,2 , on $\bar{Q}_{T}$.

Proof. Suppose that the strict inequalities of (19) hold; by Lemma 7, we have $w_{i}(x, t)>0$. Now we consider the general case. Set

$$
v_{i}=w_{i}+\varepsilon e^{K t},
$$

where $\varepsilon$ is any fixed positive constant, and $K=1+$ $\max \left\{\int_{\Omega}\left(a_{i}(x, t)+b_{i}(x, t)\right) \mathrm{d} x, i=1,2, \ldots, k\right\}$. By (19), we get, for $i=1,2, \ldots, k$,

$$
\begin{aligned}
& v_{i t}-\Delta v_{i}-\int_{\Omega}\left(a_{i}(x, t) v_{i}+b_{i}(x, t) v_{i+1}\right) \mathrm{d} x \\
& \geq \varepsilon e^{K t}\left(K-\int_{\Omega}\left(a_{i}(x, t)+b_{i}(x, t)\right) \mathrm{d} x\right)>0, \\
& \qquad(x, t) \in Q_{T}, \\
& v_{i}(x, t)-\int_{\Omega} g_{i}(x, y) v_{i}(y, t) \mathrm{d} y \quad(x, t) \in S_{T}, \\
& \geq \varepsilon e^{K t}\left(1-\int_{\Omega} g_{i}(x, y) \mathrm{d} y\right)>0, \quad x \in \Omega,
\end{aligned}
$$


Therefore, we have $v_{i}(x, t) \geq 0$ on $Q_{T}$. Letting $\varepsilon \rightarrow 0^{+}$, we get the desired result.

If the boundary condition $\int_{\Omega} g_{i}(x, y) \mathrm{d} y<1$ is not necessarily valid, we have the following result. The argument of its proof can be referred to [22, Lemma 2.2].

Lemma 9. Suppose that $a_{i j}, b_{i}, f_{i} \in C\left(\bar{Q}_{T}\right), f_{i} \geq 0, c_{i}, d_{i}$, are nonnegative and bounded in $Q_{T}, g_{i}(x, y) \geq 0$ on $\partial \Omega \times \bar{\Omega}$, $\int_{\Omega} g_{i}(x, y) \mathrm{d} y>0$ on $\partial \Omega, i=1,2, \ldots, k, j=1,2, \ldots, N$. If, for every $i=1,2, \ldots, k, w_{i} \in C^{2,1}\left(Q_{T}\right) \cap C\left(\bar{Q}_{T}\right)$ and satisfies

$$
\begin{aligned}
w_{i t}-\Delta w_{i} \geq \sum_{j=1}^{N} a_{i j} \frac{\partial w_{i}}{\partial x_{j}}+b_{i} w_{i} \\
+f_{i}(x, t) \int_{\Omega}\left(c_{i} w_{i}+d_{i} w_{i+1}\right) \mathrm{d} x, \quad(x, t) \in Q_{T}, \\
w_{i}(x, t) \geq \int_{\Omega} g_{i}(x, y) w_{i}(y, t) \mathrm{d} y, \quad(x, t) \in S_{T}, \\
w_{i}(x, 0) \geq 0, \quad x \in \Omega,
\end{aligned}
$$

where $w_{k+1}=w_{1}$, then $w_{i}(x, t) \geq 0, i=1,2$, on $\bar{Q}_{T}$.

By Lemma 9, we can easily get the following result.

Lemma 10. Let $\left(\bar{u}_{1}, \bar{u}_{2}, \ldots, \bar{u}_{k}\right)$ and $\left(\underline{u}_{1}, \underline{u}_{2}, \ldots, \underline{u}_{k}\right)$ be nonnegative upper and lower solution of system (1) on $\bar{Q}_{t}$, respectively. If one assumes that, for some $r \in\{1,2, \ldots, k\}$,

(i) $\bar{u}_{r+1}>\delta$ or $\underline{u}_{r+1}>\delta$ when $p_{r}<1$,

(ii) $\bar{u}_{r}>\delta$ or $\underline{u}_{r}>\delta$ when $q_{r}<1$,

then $\left(\bar{u}_{1}, \bar{u}_{2}, \ldots, \bar{u}_{k}\right) \geq\left(\underline{u}_{1}, \underline{u}_{2}, \ldots, \underline{u}_{k}\right)$ on $Q_{T}$.

\section{Global Existence Results}

Before proving Theorem 2, we give a global existence result for a scalar equation.

Lemma 11. Let $w_{0}(x)$ and $\varphi(x, y)$ be continuous, nonnegative functions on $\bar{\Omega}$ and $\partial \Omega \times \bar{\Omega}$, respectively, and let the nonnegative constants $\theta_{i j}$ satisfy $0<\theta_{i 1}+\theta_{i 2} \leq 1$. Then the solutions of the nonlocal problem

$$
\begin{gathered}
w_{t}-\Delta w=\sum_{i=1}^{k} w^{\theta_{i 1}}(x, t) \int_{\Omega} w^{\theta_{i 2}}(x, t) \mathrm{d} x, \quad x \in \Omega, t>0, \\
w(x, t)=\int_{\Omega} \varphi(x, y) w(y, t) \mathrm{d} y, \quad x \in \partial \Omega, t>0, \\
w(x, 0)=w_{0}(x), \quad x \in \Omega
\end{gathered}
$$

exist globally.

Proof. The augment is similar to the proof of [22, Lemma 3.1] or [21, Lemma 6]. For the reader's convenience, we complete it. It is easy to prove that there exists a positive function $\psi \epsilon$ $C^{2}(\bar{\Omega})$ such that

$$
\begin{aligned}
& \min _{\bar{\Omega}} \psi(x)>\max _{\bar{\Omega}} w_{0}^{2}(x), \\
& \psi(x) \geq \int_{\Omega} \varphi^{2}(x, y) \mathrm{d} y \int_{\Omega} \psi(y) \mathrm{d} y, \\
& x \in \partial \Omega .
\end{aligned}
$$

Let $\theta>0$ be large enough such that

$$
\begin{aligned}
& 2 \theta \min _{\bar{\Omega}} \psi(x) \\
& \geq(2 k+1) \max \left\{\max _{\bar{\Omega}}|\Delta \psi(x)|,\right. \\
&|\Omega|\left[\max _{\bar{\Omega}} \psi(x)\right]^{\left(\theta_{i 1}+\theta_{i 2}+1\right) / 2} \\
&(i=1,2, \ldots, k)|\Omega|\} .
\end{aligned}
$$

Setting $z(x, t)=e^{2 \theta t} \psi(x)$ for $(x, t) \in \Omega \times(0, \infty)$, one readily checks that

$$
\begin{gathered}
z_{t}-\Delta z \geq 2 \sum_{i=1}^{k} z^{\left(\theta_{i 1}+1\right) / 2}(x, t) \int_{\Omega} z^{\theta_{i 2} / 2}(x, t) \mathrm{d} x, \\
x \in \Omega, t>0, \\
z(x, t) \geq \int_{\Omega} \varphi^{2}(x, y) \mathrm{d} y \int_{\Omega} z(y, t) \mathrm{d} y, \quad x \in \partial \Omega, t>0, \\
z(x, 0) \geq w_{0}^{2}(x)+1, \quad x \in \Omega,
\end{gathered}
$$

Let $\bar{w}=z^{1 / 2}(x, t)$; it follows that

$$
\begin{gathered}
\bar{w}_{t}-\Delta \bar{w} \geq \sum_{i=1}^{k} \bar{w}^{\theta_{i 1}}(x, t) \int_{\Omega} \bar{w}^{\theta_{i 2}}(x, t) \mathrm{d} x, \quad x \in \Omega, t>0, \\
\bar{w}(x, t) \geq \int_{\Omega} \varphi^{2}(x, y) \mathrm{d} y \int_{\Omega} \bar{w}(y, t) \mathrm{d} y, \quad x \in \partial \Omega, t>0, \\
\bar{w}(x, 0)>w_{0}(x), \quad x \in \Omega .
\end{gathered}
$$

This implies that $\bar{w}$ is a global upper solution of (23). Clearly, 0 is a lower solution of it. So we complete the proof.

Proof of Theorem 2. By (11), we know that there exists $a_{i} \in$ $(0,1), i=1,2, \ldots, k$, such that

$$
\frac{p_{i}}{1-q_{i}} \leq \frac{a_{i}}{a_{i+1}}, \quad i=1,2, \ldots, k, a_{k+1}=a_{1} .
$$


Define $\alpha=\sum_{i=1}^{k} 1 / a_{i}$. Let $\Phi(x, y) \geq \max \left\{\varphi_{i}(x, y), i=\right.$ $1,2, \ldots, k\}$ be a continuous function defined for $(x, y) \in \partial \Omega \times$ $\bar{\Omega}$. Suppose that $z$ solves

$$
\begin{gathered}
z_{t}-\Delta z=\alpha \sum_{i=1}^{k} z^{1-a_{i}}(x, t) \int_{\Omega} z^{a_{i}}(x, t) \mathrm{d} x, \quad x \in \Omega, t>0, \\
z(x, t)=\sum_{i=1}^{k} g_{i}(x) \int_{\Omega} \Phi(x, y) z(y, t) \mathrm{d} y, \quad x \in \partial \Omega, t>0, \\
z(x, 0)=1+\sum_{i=1}^{k} u_{i, 0}^{1 / a_{i}}(x), \quad x \in \Omega,
\end{gathered}
$$

where

$$
g_{i}(x)=\left(\int_{\Omega} \Phi(x, y) \mathrm{d} y\right)^{\left(1-a_{i}\right) / a_{i}} .
$$

In view of Lemma 11, we know that $z$ is global. Moreover, $z>$ 1 in $\bar{\Omega} \times[0, \infty)$ by the maximum principle. Set $\bar{u}_{i}=z^{a_{i}}, i=$ $1,2, \ldots, k$. By (28) and (29) and using Hölder's inequality, we get

$$
\begin{aligned}
& \bar{u}_{i t}- \Delta \bar{u}_{i}-\int_{\Omega} \bar{u}_{i}^{q_{i}} \bar{u}_{i+1}^{p_{i}} \mathrm{~d} x \\
&= a_{i} z^{a_{i}-1} z_{t}-a_{i} z^{a_{i}-1} \Delta z-a_{i}\left(a_{i}-1\right)|\nabla z|^{2} \\
&-\int_{\Omega} z^{a_{i} q_{i}+a_{i+1} p_{i}} \mathrm{~d} x \\
& \geq a_{i} z^{a_{i}-1}\left(z_{t}-\Delta z\right)-\int_{\Omega} z^{a_{i}} \mathrm{~d} x \geq\left(\alpha a_{i}-1\right) \int_{\Omega} z^{a_{i}} \mathrm{~d} x \\
& \geq 0, \quad(x, t) \in Q_{T}, \\
& \bar{u}_{i}- \int_{\Omega} \varphi_{i}(x, y) \bar{u}_{i}(y, t) \mathrm{d} y \\
&= z^{a_{i}}-\int_{\Omega} \varphi_{i}(x, y) z^{a_{i}}(y, t) \mathrm{d} y \\
& \geq\left(\int_{\Omega} \varphi_{i}(x, y) \mathrm{d} y\right)^{1-a_{i}}\left(\int_{\Omega} \varphi_{i}(x, y) z(y, t) \mathrm{d} y\right)^{a_{i}} \\
&-\int_{\Omega} \varphi_{i}(x, y) z^{a_{i}}(y, t) \mathrm{d} y \\
& \geq 0, \quad(x, t) \in S_{T}, \\
& \bar{u}_{i}(x, 0) \geq u_{i, 0}(x), \quad x \in \Omega .
\end{aligned}
$$

This means that $\left(\bar{u}_{1}, \bar{u}_{2}, \ldots, \bar{u}_{k}\right)$ is a global upper solution of (1).

\section{Proof of Theorem 3. Define}

$$
\max \left\{\sup _{\partial \Omega} \int_{\Omega} \varphi_{i}(x, y) \mathrm{d} y, i=1,2, \ldots, k\right\}=\delta_{0} \in(0,1) .
$$

Let $w$ be the unique solution of the elliptic problem

$$
-\Delta w=1, \quad x \in \Omega ; \quad w=C_{0}, \quad x \in \partial \Omega .
$$

Then there exists a constant $M>0$ such that $C_{0} \leq w(x) \leq$ $C_{0}+M$ in $\bar{\Omega}$. We choose $C_{0}$ to be large enough such that

$$
\frac{1+C_{0}}{1+C_{0}+M} \geq \delta_{0} \text {. }
$$

Set $\bar{u}_{i}(x, t)=b_{i}(1+w(x))$. When $(x, t) \in S_{T}$, it follows that

$$
\begin{aligned}
\bar{u}_{i} & -\int_{\Omega} \varphi_{i}(x, y) \bar{u}_{i}(y, t) \mathrm{d} y \\
& =b_{i}\left(1+C_{0}\right)-b_{i} \int_{\Omega} \varphi_{i}(x, y)(1+w(y)) \mathrm{d} y \\
& \geq b_{i}\left[1+C_{0}-\left(1+C_{0}+M\right) \delta_{0}\right] \\
& \geq 0 .
\end{aligned}
$$

Now we investigate $(x, t) \in Q_{T}$. Set $L_{i}=\left(1+C_{0}+M\right)^{p_{i}+q_{i}}|\Omega|$ for convenience. A simple computation yields

$$
\begin{aligned}
\bar{u}_{i t} & -\Delta \bar{u}_{i}-\int_{\Omega} \bar{u}_{i}^{q_{i}} \bar{u}_{i+1}^{p_{i}} \mathrm{~d} x \\
& =b_{i}-b_{i}^{q_{i}} b_{i+1}^{p_{i}} \int_{\Omega}(1+w(x))^{p_{i}+q_{i}} \mathrm{~d} x \\
& \geq b_{i}^{q_{i}}\left(b_{i}^{1-q_{i}}-b_{i+1}^{p_{i}} L_{i}\right) .
\end{aligned}
$$

(a) If $q_{r}>1$, no matter $q_{r+1}>1$ or $q_{r+1} \leq 1$, we can choose $b_{r}$ to be small enough such that $b_{r}^{1-q_{r}} \geq b_{r+1}^{p_{r}} L_{r}$. For fixed $b_{r}$, there exist $b_{i}, i=1,2, \ldots, r-1, r+1, \ldots, k$, satisfying $b_{i}^{1-q_{i}} \geq b_{i+1}^{p_{i}} L_{i}, i=1,2, \ldots, k$. It follows that

$$
\bar{u}_{i t}-\Delta \bar{u}_{i}-\int_{\Omega} \bar{u}_{i}^{q_{i}} \bar{u}_{i+1}^{p_{i}} \mathrm{~d} x \geq 0, \quad i=1,2, \ldots, k .
$$

(b) If $q_{i} \leq 1, i=1,2, \ldots, k$ and $p_{1} p_{2} \cdots p_{k}>\left(1-q_{1}\right)(1-$ $\left.q_{2}\right) \cdots\left(1-q_{k}\right)$, we can choose $b_{1}$ to be small enough such that

$$
\begin{gathered}
b_{1}^{\left(1-q_{1}\right)\left(1-q_{2}\right) \cdots\left(1-q_{k}\right)} \\
>b_{1}^{p_{1} p_{2} \cdots p_{k}} L_{1}^{\left(1-q_{2}\right) \cdots\left(1-q_{k}\right)} L_{2}^{p_{1}\left(1-q_{3}\right) \cdots\left(1-q_{k}\right)} \\
\quad \cdots L_{k-1}^{p_{1} p_{2} \cdots p_{k-2}\left(1-q_{k-1}\right)} L_{k}^{p_{1} p_{2} \cdots p_{k-1}} .
\end{gathered}
$$

Consequently, there exist $b_{i}>0, i=2,3, \ldots, k, b_{k+1}=b_{1}$ satisfying $b_{i}^{1-q_{i}} \geq b_{i+1}^{p_{i}} L_{i}, i=1,2, \ldots, k$. Hence (37) holds too.

By (35) and (37), in any case (a) or (b), we know that the solution of (1) must be global for small data $u_{i, 0}(x) \leq b_{i}(1+$ $w(x)), i=1,2, \ldots, k$ for $x \in \Omega$.

\section{Blow-Up Results}

In this section, we assume that $(u(x, t), v(x, t))$ is a positive solution of (1) on $\bar{\Omega} \times[0, T)$, where $T$ is the maximal existence time. 
Proof of Theorem 4. We denote by $\lambda_{1}, \phi_{1}(x)$ the first eigenvalue and the corresponding eigenfunction of the linear elliptic problem:

$$
-\Delta \varphi(x)=\lambda \varphi(x), \quad x \in \Omega ; \quad \varphi(x)=0, \quad x \in \partial \Omega,
$$

and $\phi_{1}(x)$ satisfies

$$
\varphi_{1}(x)>0, \quad x \in \Omega, \quad \max _{\bar{\Omega}} \phi_{1}(x)=1
$$

Define $\gamma=\min \left\{\alpha_{i}\left(q_{i}-1\right)+\alpha_{i+1} p_{i}+1, i=1,2, \ldots, k\right\}$.

(a) If $q_{r} \geq 1$, we claim that there exist positive constants $\alpha_{i}>1, i=1,2, \ldots, k$, such that the inequality

$$
\alpha_{i}\left(q_{i}-1\right)+\alpha_{i+1} p_{i}>0
$$

holds. First, when $i=r$, (41) holds for any $\alpha_{r}, \alpha_{r+1}>1$. When $i=r+1$, if $q_{r+1} \geq 1$, (41) holds for any $\alpha_{r+2}>1$; if $q_{r+1} \leq 1$ we can choose $\alpha_{r+2}>\max \left\{1, \alpha_{r+1}\left(1-q_{r+1}\right) / p_{r+1}\right\}$. That is, (41) holds too. When $i=r-1$, if $q_{r-1} \geq 1$, (41) holds for any $\alpha_{r-1}>$ 1 ; if $q_{r-1}<1$, we can choose $1<\alpha_{r-1}<\left(\alpha_{i} p_{r-1} / 1-q_{r-1}\right)$ such that (41) holds too.

(b) If $q_{i}<1, i=1,2, \ldots, k$, and $p_{1} p_{2} \cdots p_{k}>\left(1-q_{1}\right)(1-$ $\left.q_{2}\right) \cdots\left(1-q_{k}\right)$, we can choose $\alpha_{i}>1$ such that

$$
\frac{p_{1}}{1-q_{1}}>\frac{\alpha_{1}}{\alpha_{2}}, \quad \frac{p_{2}}{1-q_{2}}>\frac{\alpha_{2}}{\alpha_{3}}, \ldots, \frac{p_{k}}{1-q_{k}}>\frac{\alpha_{k}}{\alpha_{1}}
$$

Hence (41) holds too.

Hence, for the case (a) or (b), we all have $\gamma>1$. Now let $s(t)$ be the unique solution of the ODE problem

$$
\begin{gathered}
s^{\prime}(t)=-\lambda s(t)+l s^{\gamma}(t), \quad t>0, \\
s(0)=s_{0}>1,
\end{gathered}
$$

where $l=\min \left\{\left(1 / \alpha_{i}\right) \int_{\Omega} \phi_{1}^{\alpha_{i} q_{i}+\alpha_{i+1} p_{i}}, i=1,2, \ldots, k\right\}$. Then $s(t)$ blows up in finite time $T\left(s_{0}\right)$ with $s_{0}$ being large enough.

Set

$$
\begin{array}{r}
\underline{u}_{i}=s^{\alpha_{i}}(t) \phi_{1}^{\alpha_{i}}(x), \quad(x, t) \in \bar{\Omega} \times\left[0, T\left(s_{0}\right)\right), \\
i=1,2, \ldots, k .
\end{array}
$$

We will show that $(\underline{u}, \underline{v})$ is a lower solution of problem (1). A direct computation yields

$$
\begin{aligned}
\underline{u}_{i t}- & \Delta \underline{u}_{i}-\int_{\Omega} \underline{u}_{i}^{q_{i}} \underline{u}_{i+1}^{p_{i}} \mathrm{~d} x \\
= & \alpha_{i} l s^{\alpha_{i}-1+\gamma} \phi_{1}^{\alpha_{i}}-\alpha_{i}\left(\alpha_{i}-1\right) s^{\alpha_{i}} \phi_{1}^{\alpha_{i}-2}\left|\nabla \phi_{1}\right|^{2} \\
& -\int_{\Omega} s^{\alpha_{i} q_{i}+a_{i+1} p_{i}} \phi_{1}^{\alpha_{i} q_{i}+a_{i+1} p_{i}} \mathrm{~d} x
\end{aligned}
$$

$$
\begin{aligned}
& \leq \alpha_{i} l s^{\alpha_{i}-1+\gamma}-s^{\alpha_{i} q_{i}+a_{i+1} p_{i}} \int_{\Omega} \phi_{1}{ }^{\alpha_{i} q_{i}+a_{i+1} p_{i}} \mathrm{~d} x \\
& \leq 0, \quad(x, t) \in \bar{\Omega} \times\left[0, T\left(s_{0}\right)\right), \\
\underline{u}_{i} & -\int_{\Omega} \varphi_{i}(x, y) \underline{u}_{i}(y, t) \mathrm{d} y \\
& =0-s^{\alpha_{i}}(t) \int_{\Omega} \varphi_{i}(x, y) \phi_{1}^{\alpha_{i}}(y) \mathrm{d} y \\
& \leq 0, \quad(x, t) \in \partial \Omega \times\left(0, T\left(s_{0}\right)\right) .
\end{aligned}
$$

$\left(\underline{u}_{1}, \ldots, \underline{u}_{k}\right)$ is a blowing up lower solution of (1) provided the initial data are so large that $u_{i, 0}(x) \geq s^{\alpha_{i}}(0) \phi_{1}^{\alpha_{i}}(x), i=$ $1,2, \ldots, k$ for $x \in \Omega$. We complete the proof.

Proof of Theorem 5. Since $u_{i, 0}>0$ in $\Omega, \int_{\Omega} \varphi_{r}(x, y) \mathrm{d} y>0$ on $\partial \Omega$, and

$$
u_{i, 0}(x)=\int_{\Omega} \varphi_{r}(x, y) u_{i, 0}(y) \mathrm{d} y, \quad x \in \partial \Omega,
$$

by the compatibility conditions, we have $u_{i, 0}>0$ on $\partial \Omega$. Denote by $\eta$ the positive constant such that $u_{i, 0}>\eta$ on $\bar{\Omega}$. The assumption $(H)$ implies that $\left(u_{i}\right)_{t}>0$ by the comparison principle, and in turn $u_{i}>\eta, i=1,2, \ldots, k$ on $\bar{\Omega} \times[0, T)$. Furthermore, $u_{r}$ satisfies

$$
\begin{gathered}
\left(u_{r}\right)_{t} \geq \Delta u_{r}+|\Omega| \eta^{p_{r}} u_{r}^{q_{r}}, \quad(x, t) \in Q_{T}, \\
u_{r}=\int_{\Omega} \varphi_{r}(x, y) u_{r}(y, t) \mathrm{d} y, \quad(x, t) \in S_{T}, \\
u_{r}(x, 0)=u_{r, 0}(x), \quad x \in \Omega .
\end{gathered}
$$

Let $z_{r}(t)$ be the solution of the following Cauchy problem:

$$
\begin{gathered}
z_{r}^{\prime}(t)=|\Omega| \eta^{p_{r}} z_{r}^{q_{r}}, \\
z_{r}(0)=\frac{1}{2} \eta>0 .
\end{gathered}
$$

Clearly, $z_{r}(t)$ blows up under the condition

$$
q_{r}>1 \text {. }
$$

On the other hand, since $\int_{\Omega} \varphi_{r}(x, y) \mathrm{d} y \geq 1$, by Lemma 9, we have $u_{r} \geq z_{r}$ as long as both $u_{r}$ and $z_{r}$ exist, and thus $u_{r}$ blows up for any positive initial data. The proof now is completed.

\section{Conflict of Interests}

The authors declare that there is no conflict of interests regarding the publication of this paper.

\section{Acknowledgments}

This work was supported by Natural Science Fund for Colleges and Universities in Jiangsu Province, 12KJB110018, and College Students' Innovative Projects, 2013. 


\section{References}

[1] J. Bebernes and A. Bressan, "Thermal behavior for a confined reactive gas," Journal of Differential Equations, vol. 44, no. 1, pp. 118-133, 1982.

[2] Y. Chen and H. Gao, "Asymptotic blow-up behavior for a nonlocal degenerate parabolic equation," Journal of Mathematical Analysis and Applications, vol. 330, no. 2, pp. 852-863, 2007.

[3] Y. Chen and L. Liu, "Global blow-up for a localized nonlinear parabolic equation with a nonlocal boundary condition," Journal of Mathematical Analysis and Applications, vol. 384, no. 2, pp. 421-430, 2011.

[4] W. A. Day, "A decreasing property of solutions of parabolic equations with applications to thermoelasticity," Quarterly of Applied Mathematics, vol. 40, no. 4, pp. 468-475, 1982.

[5] K. Deng, "Nonlocal nonlinearity versus global blow-up," Mathematica Applicata, vol. 8, pp. 124-129, 1995.

[6] K. Deng, M. K. Kwong, and H. A. Levine, "The influence of nonlocal nonlinearities on the long time behavior of solutions of Burgers' equation," Quarterly of Applied Mathematics, vol. 50, no. 1, pp. 173-200, 1992.

[7] W. Deng, Y. Li, and C. Xie, "Existence and nonexistence of global solutions of some nonlocal degenerate parabolic equations," Applied Mathematics Letters, vol. 16, no. 5, pp. 803808, 2003.

[8] A. Friedman, "Monotonic decay of solutions of parabolic equations with nonlocal boundary conditions," Quarterly of Applied Mathematics, vol. 44, no. 3, pp. 401-407, 1986.

[9] J. Furter and M. Grinfeld, "Local vs. non-local interactions in population dynamics," Journal of Mathematical Biology, vol. 27, no. 1, pp. 65-80, 1989.

[10] J.-S. Guo and H. W. Su, “The blow-up behavior of the solution of an integrodifferential equation," Differential and Integral Equations, vol. 5, no. 6, pp. 1237-1245, 1992.

[11] F. Li and C. Xie, "Existence and blow-up for a degenerate parabolic equation with nonlocal source," Nonlinear Analysis: Theory, Methods \& Applications, vol. 52, no. 2, pp. 523-534, 2003.

[12] H. Lu, "Global solutions and blow-up problems to a porous medium system with nonlocal sources and nonlocal boundary conditions," Mathematical Methods in the Applied Sciences, vol. 34, no. 15, pp. 1933-1944, 2011.

[13] M. Wang and Y. Wang, "Properties of positive solutions for non-local reaction-diffusion problems," Mathematical Methods in the Applied Sciences, vol. 19, no. 14, pp. 1141-1156, 1996.

[14] J. Bebernes, A. Bressan, and A. Lacey, "Total blow-up versus single point blow-up," Journal of Differential Equations, vol. 73, no. 1, pp. 30-44, 1988.

[15] J. M. Chadam, A. Peirce, and H.-M. Yin, "The blowup property of solutions to some diffusion equations with localized nonlinear reactions," Journal of Mathematical Analysis and Applications, vol. 169, no. 2, pp. 313-328, 1992.

[16] P. Souplet, "Blow-up in nonlocal reaction-diffusion equations," SIAM Journal on Mathematical Analysis, vol. 29, no. 6, pp. 13011334, 1998.

[17] P. Souplet, "Uniform blow-Up profiles and boundary behavior for diffusion equations with nonlocal nonlinear source," Journal of Differential Equations, vol. 153, no. 2, pp. 374-406, 1999.

[18] C. V. Pao, "Blowing-up of solution for a nonlocal reactiondiffusion problem in combustion theory," Journal of Mathematical Analysis and Applications, vol. 166, no. 2, pp. 591-600, 1992.
[19] Z. Lin and Y. Liu, "Uniform blowup profiles for diffusion equations with nonlocal source and nonlocal boundary," Acta Mathematica Scientia. Series B, vol. 24, no. 3, pp. 443-450, 2004.

[20] A. Gladkov and K. I. Kim, "Uniqueness and nonuniqueness for reaction-diffusion equation with nonlocal boundary condition," Advances in Mathematical Sciences and Applications, vol. 19, no. 1, pp. 39-49, 2009.

[21] L.-H. Kong and M.-X. Wang, "Global existence and blow-up of solutions to a parabolic system with nonlocal sources and boundaries," Science in China, Series A, vol. 50, no. 9, pp. 12511266, 2007.

[22] S. Zheng and L. Kong, "Roles of weight functions in a nonlinear nonlocal parabolic system," Nonlinear Analysis: Theory, Methods \& Applications, vol. 68, no. 8, pp. 2406-2416, 2008.

[23] M. Escobedo and M. A. Herrero, "A semilinear parabolic system in a bounded domain," Annali di Matematica Pura ed Applicata, vol. 165, no. 1, pp. 315-336, 1993. 


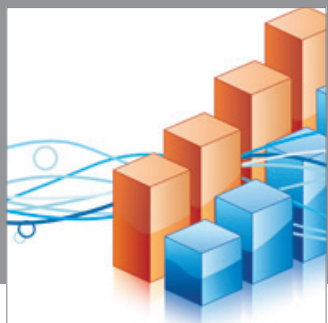

Advances in

Operations Research

mansans

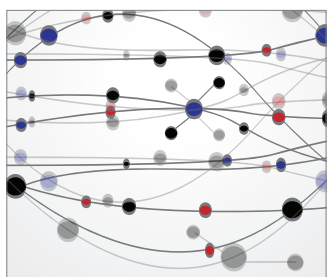

The Scientific World Journal
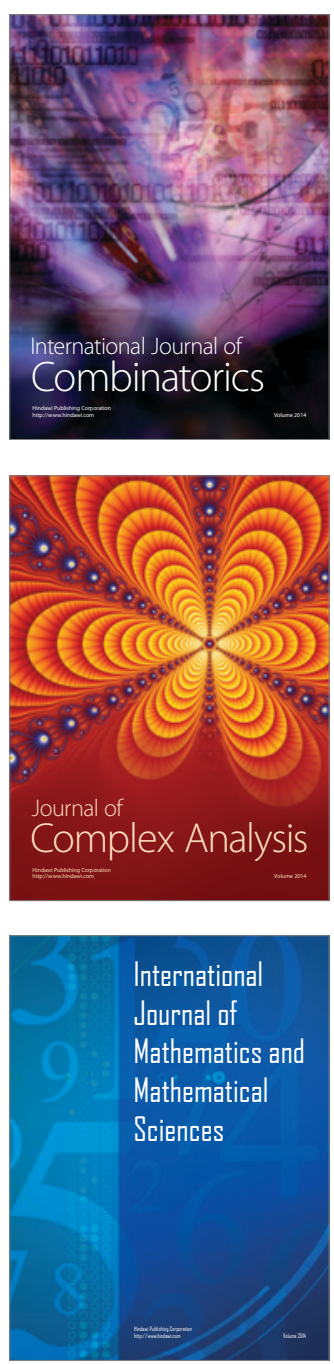
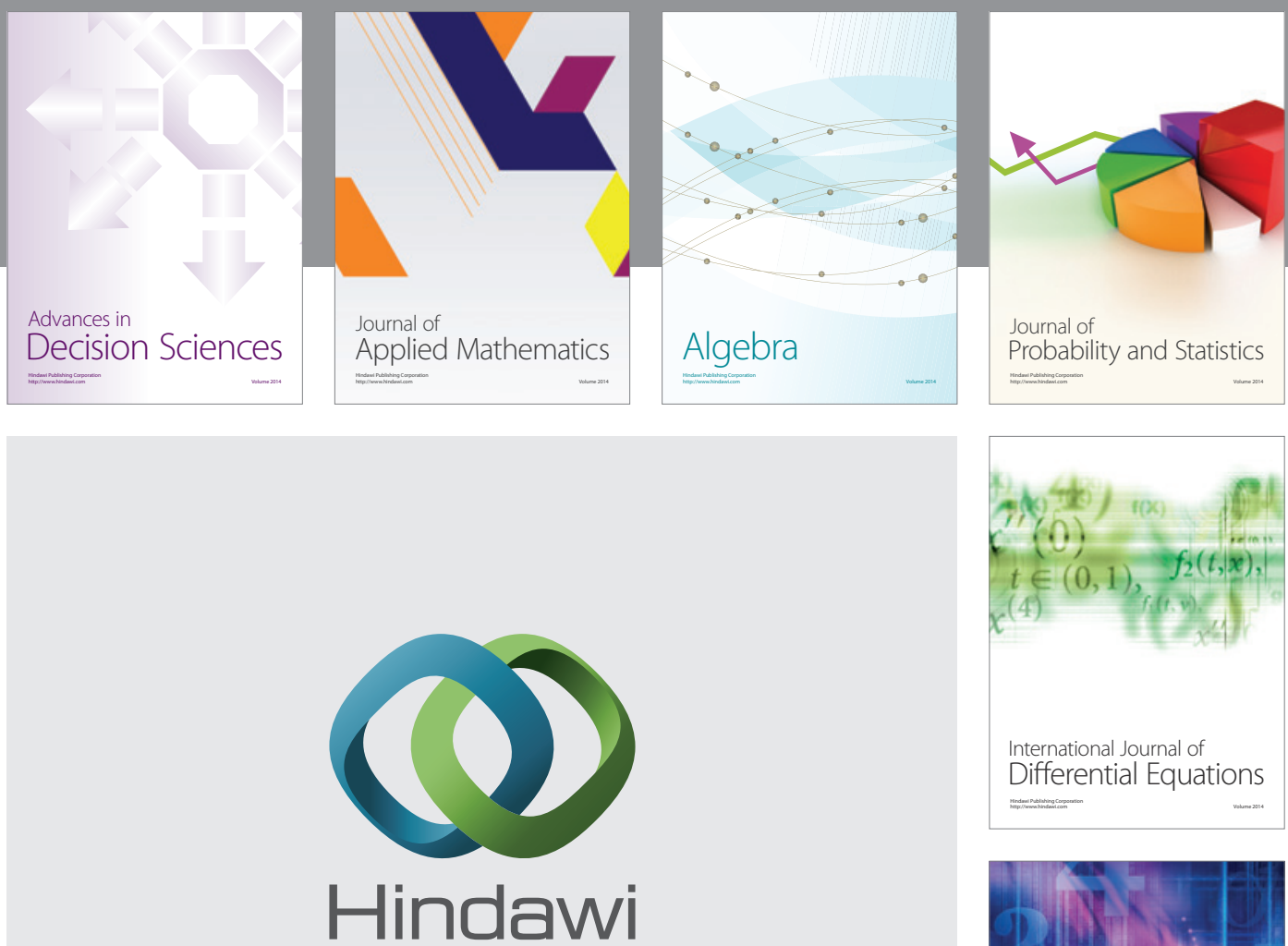

Submit your manuscripts at http://www.hindawi.com
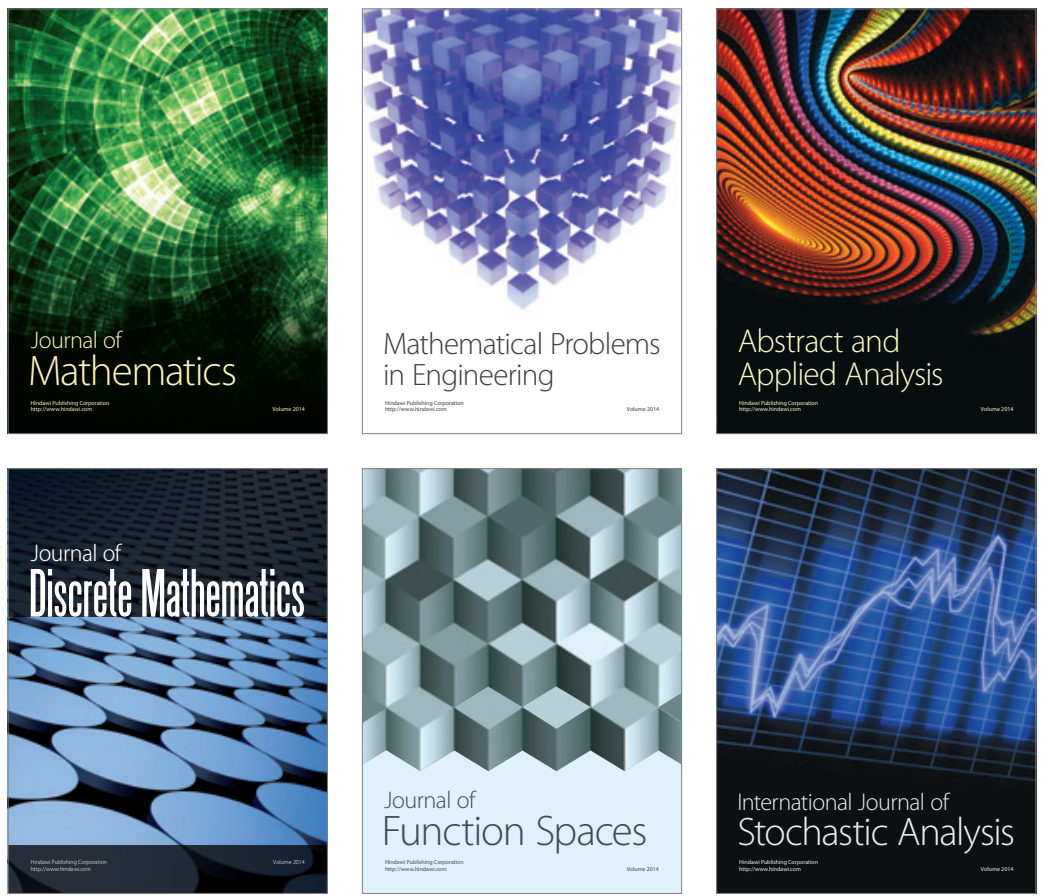

Journal of

Function Spaces

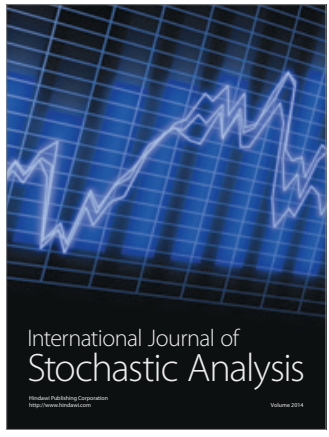

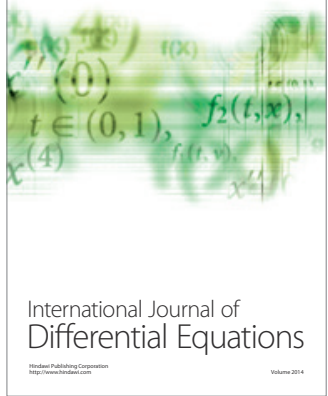
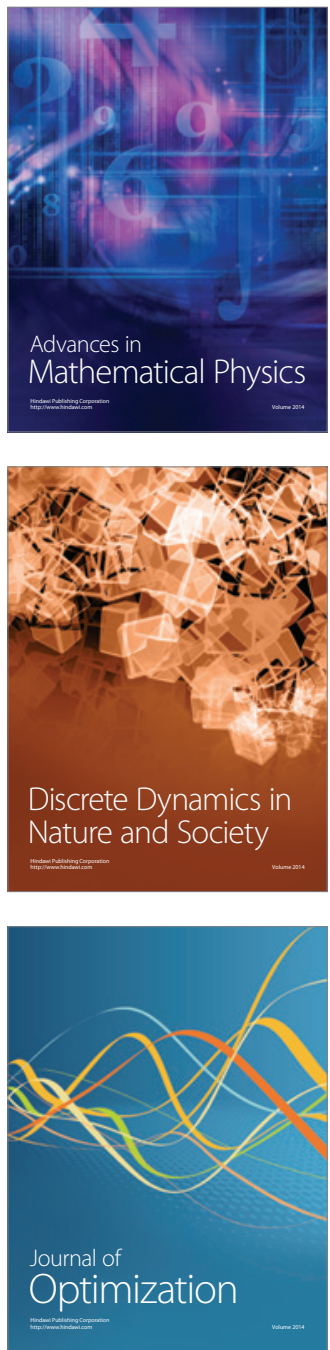